\title{
Differences in colorectal cancer surveillance epidemiology and screening in the WHO European Region
}

\author{
EMMA ALTOBELLI ${ }^{1,2}$, LEONARDO RAPACCHIETTA ${ }^{2}$, CIRO MARZILIANO ${ }^{1,2}$, GIUSEPPE CAMPAGNA ${ }^{2}$, \\ VALERIO F. PROFETA ${ }^{3}$ and ROBERTO FAGNANO ${ }^{3}$ \\ ${ }^{1}$ Department of Life, Health and Environmental Sciences, University of L'Aquila, L'Aquila I-67100; \\ ${ }^{2}$ Epidemiology and Biostatistics Unit; ${ }^{3}$ Local Health Unit 4, Teramo I-64100, Italy
}

Received May 14, 2018; Accepted November 15, 2018

DOI: $10.3892 / \mathrm{ol} .2018 .9851$

\begin{abstract}
The aim of this study was to describe the Colorectal Cancer (CRC) burden and prevention actions in 53 countries of the World Health Organization (WHO) European Region (ER). Multiple correspondence analysis was applied to examine the association among the following variables: Measures of occurrence; type of screening programme; existence of cancer registries; data quality and; and gross national income (GNI) level. The study demonstrated clear differences according to GNI: low-middle income (LMI) countries show low mortality rates and unorganized screening programme; upper-middle income (UMI) countries show no test offered, incomplete or absent data mortality, and low quality of the method used to estimate incidence and mortality rates; high income (HI) countries show high mortality rates, test offered (FOBT and colonoscopy), the existence of a national registry, screening population-based, insurance of payment policy, and high quality of the method used to estimate incidence and mortality rates. HI countries reflect a strong interest in epidemiological monitoring and produce accurate indicators of disease occurrence. On the other hand, surveillance strategies need to be improved in UMI and LMI countries: As national vital statistics are unavailable, partial or inaccurate, the coverage and completeness of the mortality data are frequently poor, there is a less efficient general organization. In conclusion, it is important to underline that the resources available (as measured by GNI) appear to be major factors in the Colorectal Cancer Surveillance Epidemiology and Screening in the WHO European Region.
\end{abstract}

Correspondence to: Professor Emma Altobelli, Department of Life, Health and Environmental Sciences, University of L'Aquila, 1 Piazzale Salvatore Tommasi, L'Aquila I-67100, Italy

E-mail: emma.altobelli@cc.univaq.it

Key words: colorectal cancer, prevention/screening programmes, income level, correspondence analysis, World Health Organization European Region

\section{Introduction}

In the World Health Organization (WHO) European Region (ER), colorectal cancer (CRC) is the first tumour by incidence, with 471,000 new cases each year and a mean mortality rate of 28.2 per 100,000 Population (1). Incidence varies, peaking in central European States and showing the lowest rates in some Mediterranean States (2). CRC incidence and mortality in a population are also related to changes in the prevalence of some modifiable risk factors such as smoking, alcohol consumption, and diet. According to epidemiological evidence, the risk of developing CRC increases in relation to lifestyle (i.e. consumption of red meat, increased alcohol consumption, etc.) (3). In addition to primary prevention, early detection and improved diagnosis and treatment of symptomatic disease are potential factors that contribute to decreasing CRC incidence and mortality.

Randomized controlled trials (RCT) have shown that screening is associated with a reduction in mortality (4). Furthermore, CRC screening based on stool testing (FOBT test) and flexible sigmoidoscopy has reduced CRC-related mortality respectively by 16 and $22-31 \%(5,6)$. According to cost-effectiveness studies, CRC screening is cost-effective compared with no screening (7).

Screening for CRC is a complex process that includes the initial screening test as well as follow-up diagnostic tests if needed. Compared with spontaneous screening (also called opportunistic), organized screening involves a greater focus on the quality of the screening process, including follow-up (8). According to Karsa and colleagues (9), at least 10 years are needed to plan and organize a screening programme, and still longer to assess the impact of a population-based (PB) programme in a country. After a European Parliament resolution invited Member States (MS) to adopt cancer screening programmes in 2010, several European countries have introduced organized, PB CRC screening programmes and others are planning to do so $(1,2)$. Moreover, high-quality cancer screening is nearly always available in countries wealth and gross national income (GNI) level (10). Most high GNI countries in Europe are Members States (MS) of the European Union (EU). The aim of this study was to describe factors that correlation with CRC burden and prevention actions in WHO European Region. 


\section{Materials and methods}

GNI. According to the World Bank, economies can be divided into low income (LI), lower-middle income (LMI), upper-middle income (UMI), and high income (HI) in relation to GNI per capita (10). In this study, the $53 \mathrm{WHO}$, ER countries were thus divided into: LMI, \$1,026-4,035 (Armenia, Georgia, Kyrgyzstan, Moldova, Tajikistan, Ukraine and Uzbekistan); UMI, \$4,036-12,475 [Albania, Azerbaijan, Belarus, Bosnia and Herzegovina, Bulgaria, Kazakhstan, FYR Macedonia (FM), Hungary, Montenegro, Romania, Serbia, Turkey, Turkmenistan]; and HI, \$12,476 (Austria, Belgium, Czech Republic, Denmark, Estonia, Finland, France, Germany, Greece, Iceland, Ireland, Israel, Italy, Luxembourg, Norway, Poland, Portugal, Slovakia, Slovenia, Spain, Sweden, Switzerland, The Netherlands, and United Kingdom, Andorra, Croatia, Cyprus, Malta, Monaco, Latvia, Lithuania, Russian Federation, and San Marino) (World Bank Country and Lending Groups 2016) (Table I).

Sources of WHO European Epidemiological Data. The main data source, the GLOBOCAN 2012 website of the International Agency for Research on Cancer (IARC), provides access to several databases that allow assessing the impact of CRC in 184 countries or territories in the world (1).

These data were supplemented using the literature, ministerial web pages of individual countries, WHO web and Europe EU pages, World Bank Open Data Web pages, and the World Cancer Registry, X edition.

The quality of epidemiological data of each WHO ER country was evaluated (11). All analyzed disaggregated data are reported in Table II.

It is underlined that Andorra, Monaco, and San Marino were not included in the analysis because no data were reported.

Statistical analysis. Clusters were obtained using ward-linkage clustering analysis. In cluster analysis incidence and life expectancy data were included and we obtained 4 groups of countries (clusters) consisting of values close to each other of incidence and life expectancy.

The object of Multiple Correspondence Analysis (MCA) is to analyse categorical/categorized data that are transformed into cross tables and to demonstrate the results in a graphical manner. Multiple Correspondence Analysis (MCA) is a powerful descriptive statistical technique for handling larger, more complex datasets.

The dimensions can be interpreted in terms of distances: The more a variable (i.e. low mortality) is placed in the Cartesian plane far from the origin of the axis, the more it has a strong discriminating power and therefore characterizing the analysis. On the contrary, the more a variable (i.e. FOBT test) takes on a value close to the origin of the axis, the less it will have a discriminating value. Multiple Correspondence Analysis was applied to examine the association among the following variables: Clusters, GNI level (LMI, UMI and HI); type of CRC screening programme in country (coverage national/non-national; spontaneous/organized) $(1,12)$; existence of cancer registries; payment policies (insurance co-payment, free of charge); tests offered (FOBT and colonoscopy, only FOBT, only colonoscopy, no test); mortality and data quality. The latter measures included the availability of incidence data, the availability of mortality data, the methods adopted to estimate incidence rates, and the methods used to estimate mortality rates. These variables were coded as ordinal or nominal or dummy variables, as appropriate, and incorporated into the model.

Data quality was grouped and defined according to: i) The availability of incidence data (three categories): 'High quality', 'medium quality', and 'low quality' $(11,13)$; ii) The availability of mortality data (three categories): 'High/medium', 'low' and 'incomplete or absent' $(11,13)$; iii) the quality of the method adopted to estimate incidence rates (three categories): 'High'; 'medium' and 'low' (11,13); iv) the quality of the method used to estimate mortality rates (three categories): 'High', 'medium' and 'low' $(11,13)$. SPSS (version 22) and Minitab (version 18.1.0) software were used for statistical analysis.

\section{Results}

Cluster analysis. The cluster analysis has identified four clusters including 50 out of 53 WHO ER countries. The results are represented in Fig. 1.

Cluster 1 includes 11 countries: Albania, Georgia, Greece, Armenia, Bosnia and Herzegovina, Turkey, Azerbaijan, Kyrgyzstan, Tajikistan, Uzbekistan and Turkmenistan. Respect to all countries of WHO ER this cluster grouped 7/11 countries with the lowest incidence rates, and 5/8 countries with the lowest mortality rates.

Cluster 2 includes 18 countries: Austria, Cyprus, Finland, Estonia, Poland, Montenegro, Romania, Bulgaria, Serbia, Croatia, Belarus, Latvia, FYR Macedonia [FM], Lithuania, Kazakhstan, Ukraine, Russian Federation, Republic of Moldova. This cluster includes countries with high income (Austria, Croatia, Cyprus, Estonia, Finland, Latvia, Lithuania, Poland and Russian Federation), while East-European and Asian countries (Belarus, Bulgaria, FRY of Macedonia, Montenegro, Romania, Serbia and Kazakhstan) have upper-middle income. Republic of Moldova and Ukraine have low-middle income.

Cluster 3 includes 10 countries: Belgium, Slovenia, Ireland, Israel, Czech Republic, Denmark, The Netherlands, Norway, Hungary, Slovakia; countries with the highest incidence rates. This cluster includes countries with high income, except for Hungary with upper-middle income.

Cluster 4 includes 11 countries: France, United Kingdom, Iceland, Sweden, Switzerland, Germany, Luxembourg, Malta, Portugal, Italy, Spain; countries with the highest life expectancy. The cluster includes only countries with high income.

Multiple correspondence analysis. The results of MCA are represented in Fig. 2 (object scores plot). The data provided two dimensions with values that explain $78 \%$ of the variance: Dimension $1=0.51$ and dimension $2=0.27$. The first dimension is related to the following variables: Cluster, test offered, GNI level, availability of incidence data and the quality of the method applied to estimate incidence and mortality; the second dimension is related to the availability of mortality data and the quality of the method applied to estimate mortality. 


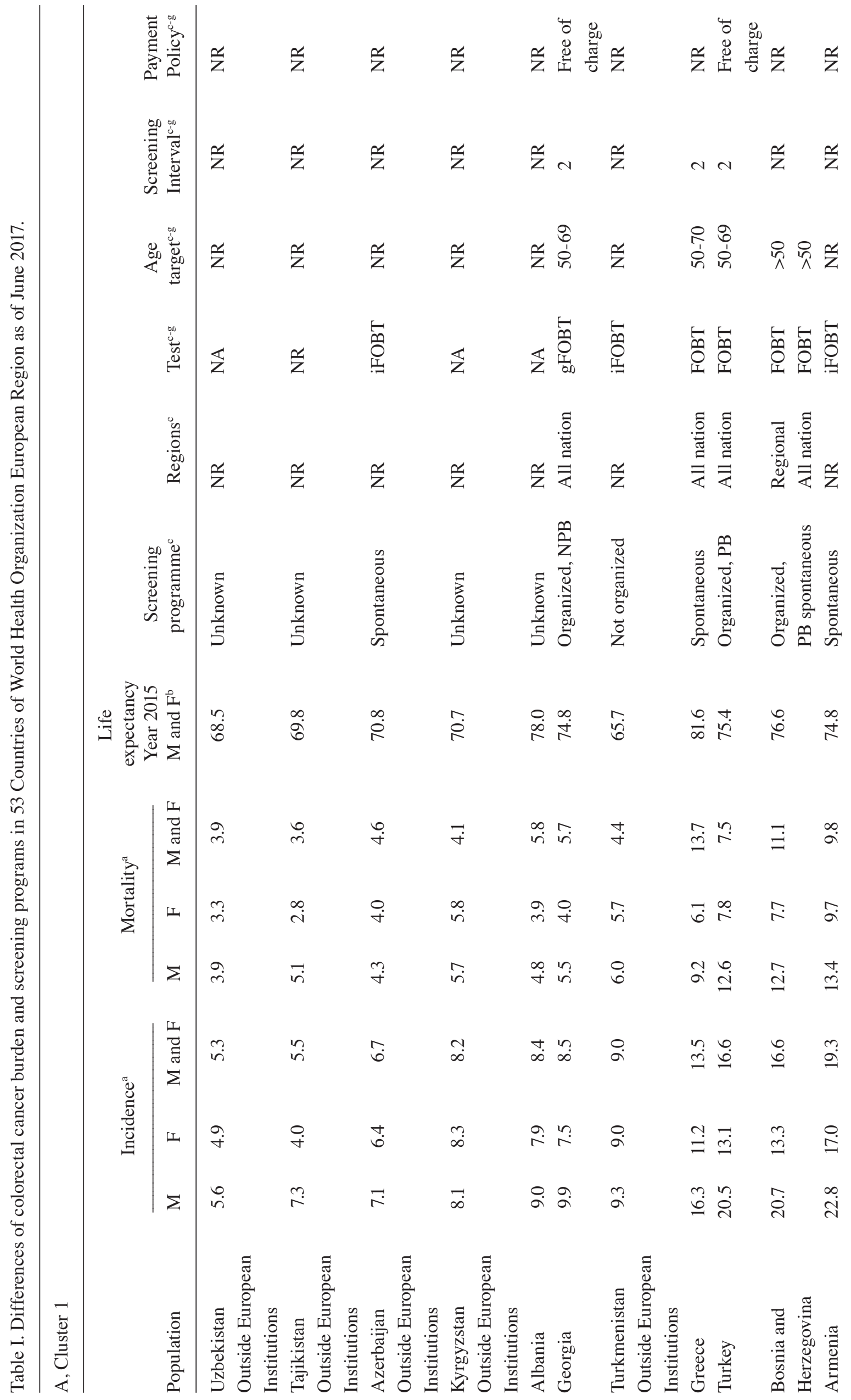


空:

它

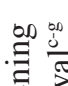

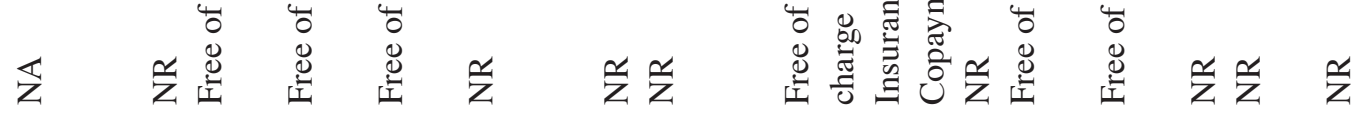

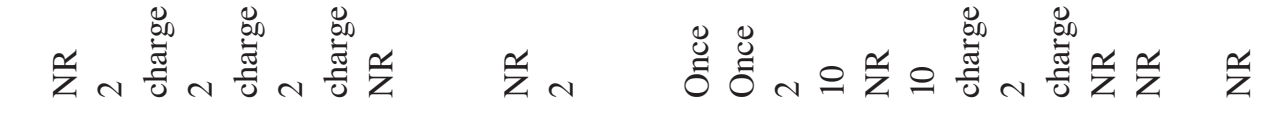

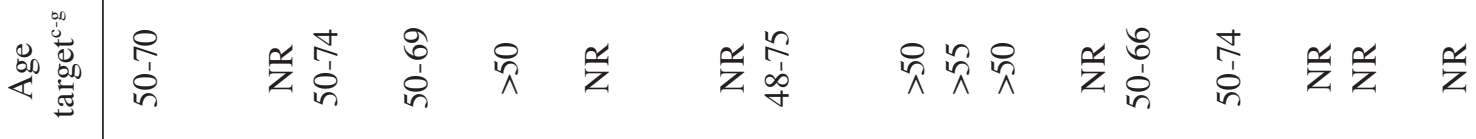

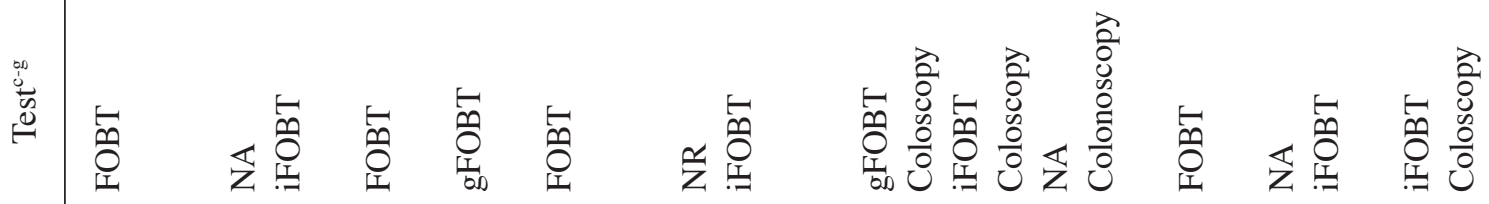

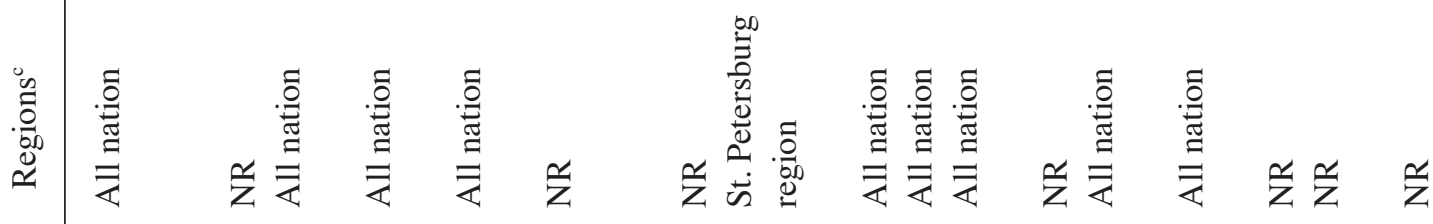

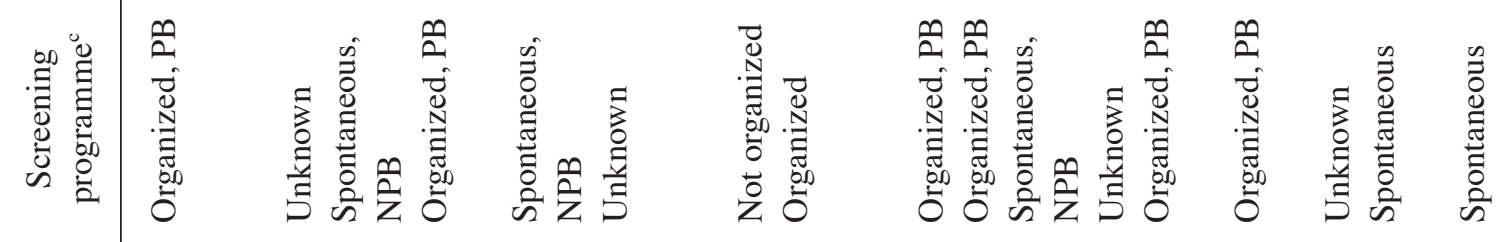

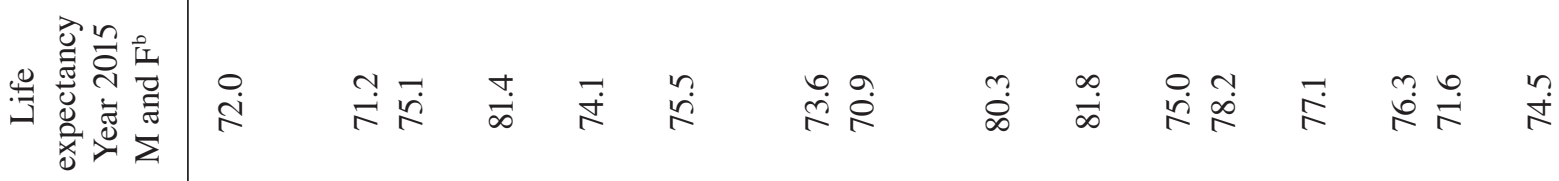

厗|

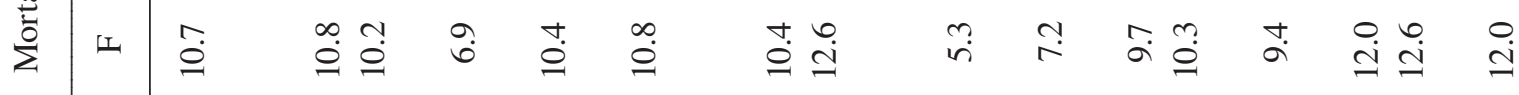

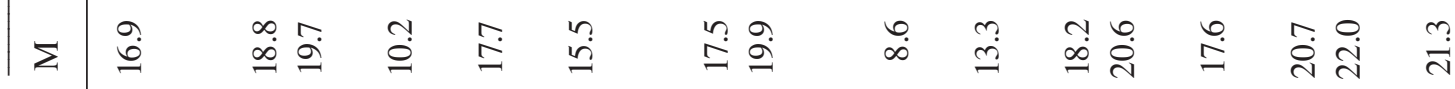

|

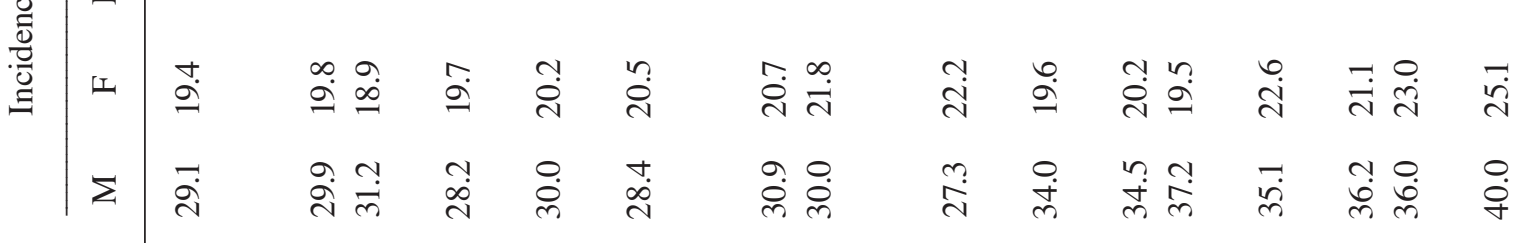



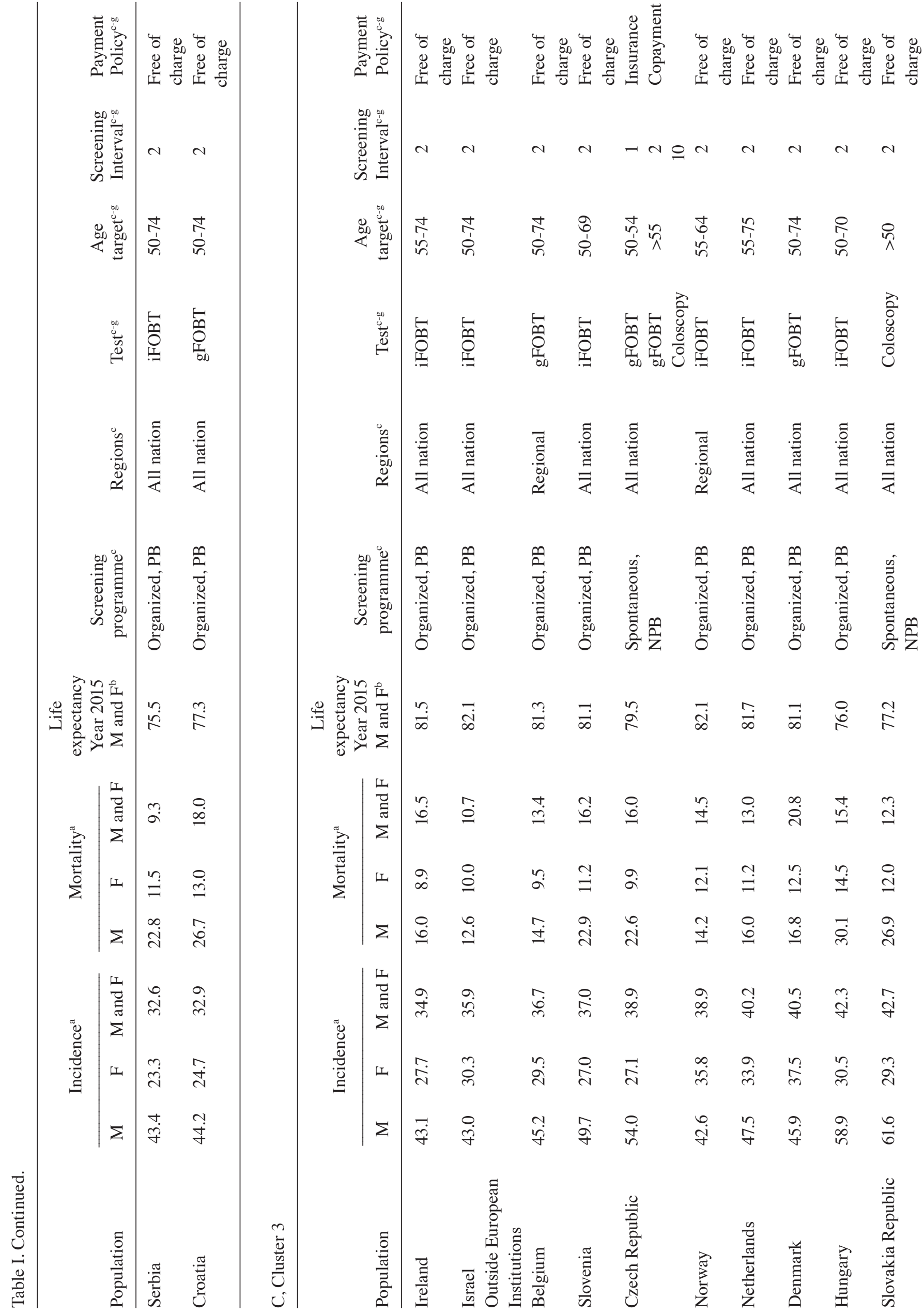


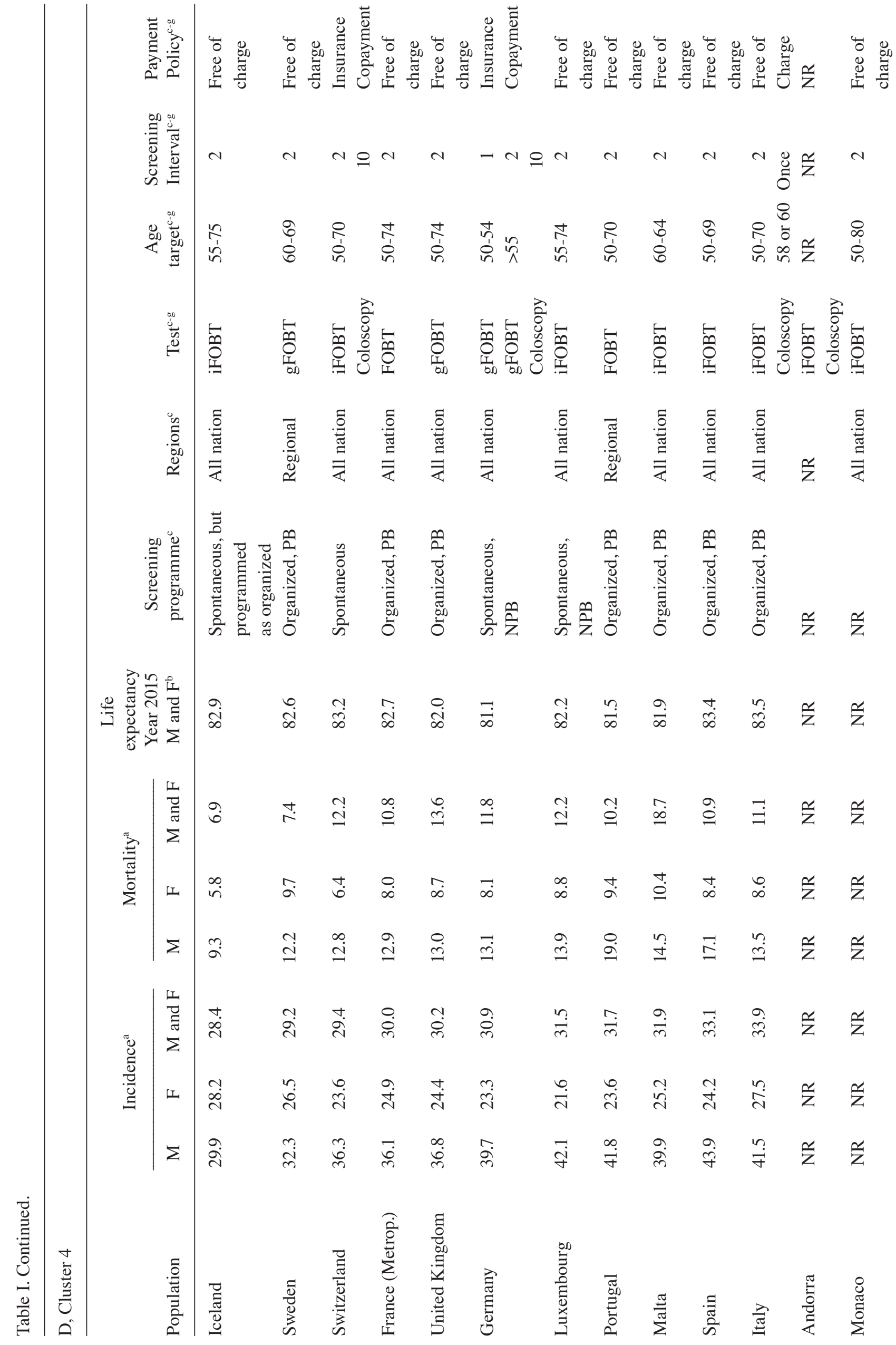


Fig. 2 is subdivided in four quadrants whose numbering is counter clockwise. In the first quadrant (upper right), the modality of variables found are: Low/medium mortality rates, medium quality incidence data, incomplete/low or absent quality mortality data and low-quality of methods applied to estimate mortality, UM income, no test offered. The modality of variables found in the second quadrant (upper left) are: Clusters 2 and 4, medium/high mortality rates, medium quality methods to estimate incidence, no-national register, population-based screening, organized screening, FOBT as test offered and free of charge. The third quadrant (lower left) includes: Cluster 3, high mortality rates, $\mathrm{H}$ income, tests offered (colonoscopy, FOBT and colonoscopy), the existence of a national registry, population-based screening, high-quality availability of incidence data, high-quality methods used to estimate incidence and mortality rates, medium/high-quality availability of mortality data, national screening, payment of insurance policy.

The modality of variables found in the fourth quadrant (lower right) includes: Cluster 1, LM income, no organized screening programme, low mortality rates, low quality of the method used to estimate incidence, medium quality of the method used to estimate mortality data, low quality availability of incidence data.

\section{Discussion}

Cancer mortality rates are mounting worldwide, and since 1990 cancer has moved up from third to second place among the causes of death, after cardiovascular disease (14). This rising trend is related to a number of factors that include population aging, high demographic growth, reduced mortality from other causes, and higher cancer rates induced in some populations by the growth of some risk factors-like tobacco smoking and Westernization of lifestyles (e.g. the diet) in low- and medium-income countries (15). It is thus critical for governments to optimise their limited resources to improve prevention, early diagnosis and treatment. This can be done using national and regional cancer burden figures. Comparison of occurrence data over time allows monitoring of the interventions adopted and the assessment of their effectiveness and the necessary eventual changes. Indeed, the reduction in mortality rates in several developed countries has been achieved through a complex combination of improvements in primary prevention, early diagnosis, screening and treatment (16). The screening programmes for early diagnosis of breast, cervical, and colorectal cancer have gotten better considerably. In Europe, a number of countries have improved their screening programmes, others have introduced the and others will do so in the near future $(2,13,17-19)$.

The most common causes of cancer death in HI countries are lung, colorectal, breast (in women) and pancreas cancer, which together accounted for $48 \%$ of all deaths in 2012 (1). The most frequent causes in LMI countries are lung, breast and colorectal cancer, which together account for around half of cancer deaths (1). CRC is the second-ranking cause of cancer death by incidence and mortality in developed countries and the fourth in developing countries (20). Approximately $45 \%$ of CRC patients die despite treatment (13), whose cost places a heavy strain on resources. Although it is well established 
Table II. Epidemiological data quality in each Country of World Health Organization European Region.

\section{A, Cluster 1}

\begin{tabular}{|c|c|c|c|c|}
\hline \multirow[b]{3}{*}{ Country } & \multicolumn{4}{|c|}{ Quality of data } \\
\hline & \multicolumn{2}{|c|}{ Data source } & \multicolumn{2}{|c|}{ Methods } \\
\hline & Incidence $^{a}$ & Mortality $^{\mathrm{a}}$ & Incidence $^{\mathrm{a}}$ & Mortality \\
\hline Uzbekistan Outside European Institutions & G & 2 & 5 & 2 \\
\hline Tajikistan Outside European Institutions & G & 3 & 5 & 2 \\
\hline Azerbaijan Outside European Institutions & G & 2 & 5 & 2 \\
\hline Kyrgyzstan Outside European Institutions & G & 2 & 5 & 1 \\
\hline Albania & $\mathrm{G}$ & 3 & 4 & 1 \\
\hline Georgia & G & 2 & 5 & 2 \\
\hline Turkmenistan Outside European Institutions & G & 2 & 5 & 1 \\
\hline Greece & G & 3 & 4 & 1 \\
\hline Turkey & $\mathrm{C}$ & 6 & 6 & 5 \\
\hline Bosnia and Herzegovina & $\mathrm{D}$ & 5 & 2 & 2 \\
\hline Armenia & G & 3 & 5 & 2 \\
\hline
\end{tabular}

B, Cluster 2

\begin{tabular}{|c|c|c|c|c|}
\hline \multirow[b]{3}{*}{ Country } & \multicolumn{4}{|c|}{ Quality of data } \\
\hline & \multicolumn{2}{|c|}{ Data source } & \multicolumn{2}{|c|}{ Methods } \\
\hline & Incidence $^{\mathrm{a}}$ & Mortality $^{\mathrm{a}}$ & Incidence $^{\mathrm{a}}$ & Mortality $^{\mathrm{b}}$ \\
\hline Kazakhstan Outside European Institutions & $\mathrm{G}$ & 2 & 5 & 2 \\
\hline Ukraine & A & 2 & 2 & 2 \\
\hline Lithuania & A & 1 & 1 & 1 \\
\hline Finland & A & 1 & 1 & 1 \\
\hline Latvia & A & 1 & 1 & 1 \\
\hline FRY of Macedonia & $\mathrm{G}$ & 3 & 4 & 1 \\
\hline Belarus Outside European Institutions & A & 2 & 1 & 2 \\
\hline Russian Federation Outside European Institutions & $\mathrm{D}$ & 2 & 1 & 1 \\
\hline Cyprus & A & 3 & 2 & 2 \\
\hline Austria & A & 2 & 1 & 1 \\
\hline Romania & $\mathrm{E}$ & 1 & 4 & 1 \\
\hline Poland & $\mathrm{C}$ & 3 & 3 & 1 \\
\hline Estonia & A & 1 & 1 & 1 \\
\hline Montenegro & G & 6 & 9 & 6 \\
\hline Republic of Moldova & A & 2 & 1 & 1 \\
\hline Serbia & $\mathrm{B}$ & 2 & 4 & 1 \\
\hline Bulgaria & A & 2 & 1 & 1 \\
\hline Croatia & A & 2 & 1 & 1 \\
\hline
\end{tabular}

C, Cluster 3

\begin{tabular}{|c|c|c|c|c|}
\hline \multirow[b]{3}{*}{ Country } & \multicolumn{4}{|c|}{ Quality of data } \\
\hline & \multicolumn{2}{|c|}{ Data source } & \multicolumn{2}{|c|}{ Methods } \\
\hline & Incidence $^{a}$ & Mortality $^{\mathrm{a}}$ & Incidence $^{a}$ & Mortality \\
\hline Ireland & A & 1 & 1 & 1 \\
\hline Israel Outside European Institutions & A & 2 & 1 & 1 \\
\hline
\end{tabular}


Table II. Continued.

\begin{tabular}{|c|c|c|c|c|}
\hline \multirow[b]{3}{*}{ Country } & \multicolumn{4}{|c|}{ Quality of data } \\
\hline & \multicolumn{2}{|c|}{ Data source } & \multicolumn{2}{|c|}{ Methods } \\
\hline & Incidence $^{a}$ & Mortality $^{\mathrm{a}}$ & Incidence $^{\mathrm{a}}$ & Mortality $^{\mathrm{b}}$ \\
\hline Belgium & A & 2 & 2 & 2 \\
\hline Slovenia & A & 1 & 1 & 1 \\
\hline Czech Republic & A & 2 & 1 & 1 \\
\hline Norway & A & 2 & 1 & 1 \\
\hline Netherlands & A & 2 & 1 & 1 \\
\hline Denmark & A & 2 & 1 & 1 \\
\hline Hungary & G & 1 & 4 & 1 \\
\hline Slovakia Republic & A & 1 & 1 & 1 \\
\hline
\end{tabular}

D, Cluster 4

\begin{tabular}{|c|c|c|c|c|}
\hline \multirow[b]{3}{*}{ Country } & \multicolumn{4}{|c|}{ Quality of data } \\
\hline & \multicolumn{2}{|c|}{ Data source } & \multicolumn{2}{|c|}{ Methods } \\
\hline & Incidence $^{\mathrm{a}}$ & Mortality $^{\mathrm{a}}$ & Incidence $^{\mathrm{a}}$ Mortality ${ }^{\mathrm{b}}$ & \\
\hline Iceland & A & 1 & 1 & 1 \\
\hline Sweden & A & 2 & 3 & 1 \\
\hline Switzerland & $\mathrm{B}$ & 2 & 3 & 1 \\
\hline France (Metrop.) & B & 2 & 3 & 1 \\
\hline United Kingdom & A & 1 & 1 & 1 \\
\hline Germany & $\mathrm{B}$ & 2 & 1 & 1 \\
\hline Luxembourg & $\mathrm{D}$ & 2 & 4 & 1 \\
\hline Portugal & $\mathrm{C}$ & 3 & 4 & 1 \\
\hline Malta & A & 1 & 1 & 1 \\
\hline Spain & $\mathrm{B}$ & 2 & 3 & 1 \\
\hline Italy & B & 2 & 3 & 1 \\
\hline Andorra & NR & NR & NR & NR \\
\hline Monaco & NR & NR & NR & NR \\
\hline San Marino & NR & NR & NR & NR \\
\hline
\end{tabular}

${ }^{\mathrm{a}}$ Quality assessment of Epidemiological data source and methods according to Mathers et al (2005). ${ }^{\mathrm{b}}$ Forman D, Bray F, Brewster DH, Gombe Mbalawa C, Kohler B, Piñeros M, Steliarova-Foucher E, Swaminathan R and Ferlay J. Data available from Cancer Incidence in Five Continents Vol. X Scientific Publication No. 164, IARC. NR, Not Reported.

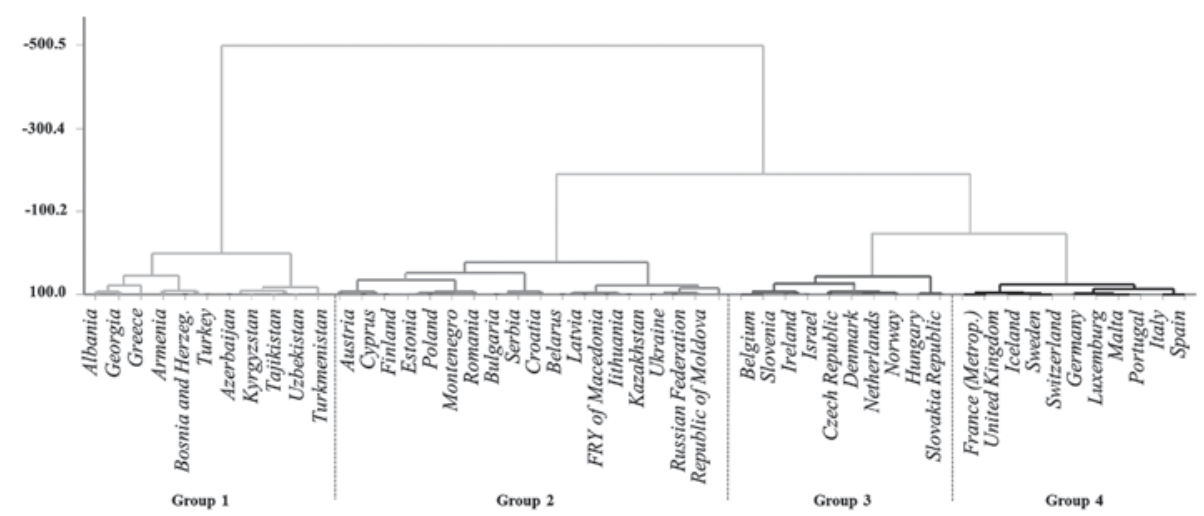

Figure 1. Picture of countries based on cluster analysis results. 


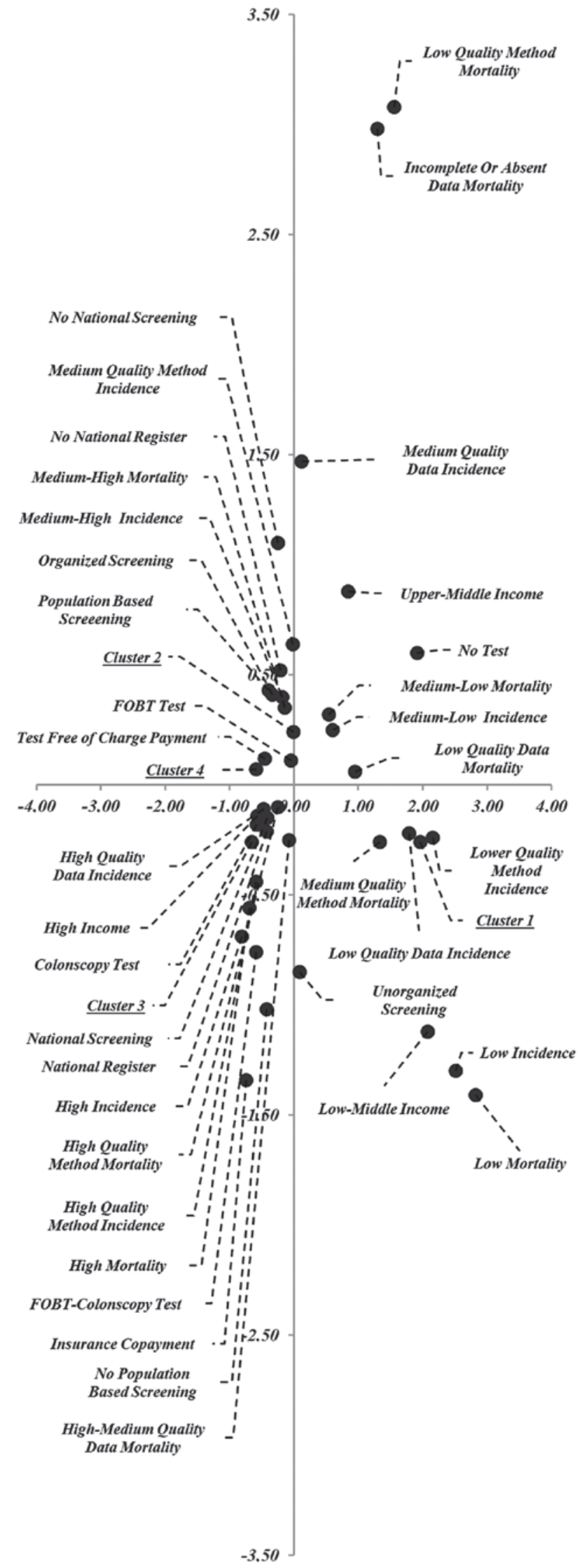

Figure 2. Association among variables included in Multiple Correspondence Analysis.

that CRC generally develops from a precursor lesion, the adenomatous polyp, and that progression to invasive cancer takes years $(21,22)$, CRC screening is currently offered to a small proportion of the target population in nearly all countries in the world, regardless of their GNI level (23). This is confirmed by the data of the 53 WHO ER countries (Table I). The variables found in the quadrant occupied by HI countries include nationwide, organized, PB screening, high incidence and mortality rates, high-quality availability of incidence and mortality data, and high quality of the method adopted to estimate incidence and mortality rates; these features form a clear pattern that reflects a strong interest in epidemiological monitoring and produces accurate indicators of disease occurrence. It should also be stressed that PB screening essentially aims at covering all the individuals at risk in a given time interval. UMI and LM countries have a less efficient general organization, and the proportion of organized programmes is low in the former while programmes are often absent in the latter. In general, CRC screening has been demonstrated to be cost-effective compared with no screening (24), also in countries with limited resources (25). Surveillance strategies also need to be improved in UMI and LM countries: Since national vital statistics are unavailable, partial or inaccurate, the coverage and completeness of the mortality data are frequently poor. A high-quality data could be associated to high-quality sources. The adoption of accurate methods to estimate incidence and mortality. Is required by cancer registries and PB screening. It is useful to underline that high-quality occurrence data are important to understand cancer trends and to implement surveillance strategies. Moreover, data on the type of screening organization, target population, rounds and tests administered are not available for the majority of LM WHO ER countries (Table I). It has been established that poor quality healthcare is associated with under screening, poor quality of screening, inappropriate use of resources, and poor follow-up of individuals who test positive on screening. In 2010, the IARC published the European Guidelines for Quality Assurance in CRC screening (26), where participation (at least 65\%) (27), follow-up, and cancer detection rates are key factors in screening performance.

The present findings are in line with the current literature in highlighting a disparity between HI and LMI areas also in relation to the population covered by cancer registries (28). This reflects on the one hand the scarce importance attributed to registries and the lack of resources allocated for their institution. However, some recent changes in priorities have been emerging in the use (and shift) of national resources allocated for cancer screening (29).

Analysis of the factors reviewed above highlighted broad variations among national CRC screening practices in the WHO ER, and prompt some reflections. First of all, high-quality occurrence data are essential to understand cancer trends and devise control strategies; critically, screening measures should always be specified both to enable comparisons among countries and to try and improve screening quality. The poor quality of the availability of incidence and mortality data and the poor quality of the detection method could result in underestimation of the respective rates. Estimates should thus be taken with caution, since a steep, unexpected increase in the frequency of cancer cases is a possible future scenario that would have repercussions on clinical practice. Secondly, the countries with the lowest GNI should use their resources 
to implement organized screening programmes also in view of the Westernization of lifestyle and of population aging, to improve patients' quality of life and survival and reduce their impact and burden on healthcare resources and facilities. In the third place, healthcare professionals should all collaborate in promoting changes in resource allocation by politicians, since healthcare investments require medical personnel, and good monitoring, evaluation, and quality control systems. HI countries that still lack an organized screening programme should urgently set them up (Greece, Austria, Lithuania, Latvia, Czech Republic, Slovakia, Switzerland, Germany and Luxemburg). Finally, small communities lacking specialized staff and laboratories and the economic resources to set up screening programmes could rely on nearby centres or regions having the resources and facilities for quality screening. In addition, provision of cancer screening might be related directly to the type of health care scheme and coverage. Furthermore, effectiveness in screening for other cancers may be used as benchmark data.

The success of preventive policies depends not only on the scientific evidence they produce, but also on the awareness of the participants in this highly complex process. In addition, it is important to underline that the resources (as measured by GNI) available appear to be major factors in the quality of surveillance epidemiology, and prevention programmes.

\section{Acknowledgements}

Not applicable.

\section{Funding}

No funding was received.

\section{Availability of data and materials}

The datasets used and/or analyzed during the current study are available from the corresponding author on reasonable request.

\section{Authors' contributions}

EA made substantial contributions to the conception and design of the present study, conducted the literature review, developed the statistical analysis, and drafted, edited and critically revised the manuscript. LR contributed to the literature search and built the database. GC participated in the literature search and acquired the data. RF and VFP participated in data acquisition. CM participated in data acquisition. All authors read and approved the final version of the manuscript.

\section{Ethics approval and consent to participate}

Not applicable.

\section{Patient consent for publication}

Not applicable.

\section{Competing interests}

The authors declare that they have no competing interests.

\section{References}

1. Ferlay J, Soerjomataram I, Ervik M, Dikshit R, Eser S, Mathers C, Rebelo M, Parkin DM, Forman D and Bray F: GLOBOCAN 2012 v1.0, cancer incidence and mortality worldwide: IARC cancer base no. 11. Lyon, France: International Agency for Research on Cancer; 2014. http://globocan.iarc.fr. Accessed March 23, 2018.

2. Altobelli E, Lattanzi A, Paduano R, Varassi G and di Orio F: Colorectal cancer prevention in Europe: Burden of disease and status of screening programs. Prev Med 62: 132-141, 2014.

3. Johnson CM, Wei C, Ensor JE, Smolenski DJ, Amos CI, Levin B and Berry DA: Meta-analyses of colorectal cancer risk factors. Cancer Causes Control 24: 1207-1222, 2013.

4. Fitzpatrick-Lewis D, Ali MU, Warren R, Kenny M, Sherifali D and Raina P: Screening for colorectal cancer: A systematic review and meta-analysis. Clin Colorectal Cancer 15: 298-313, 2016.

5. Heresbach D, Manfredi S, D'halluin PN, Bretagne JF and Branger B: Review in depth and meta-analysis of controlled trials on colorectal cancer screening by faecal occult blood test. Eur J Gastroenterol Hepatol 18: 427-433, 2006.

6. Schoen RE, Pinsky PF, Weissfeld JL, Yokochi LA, Church T, Laiyemo AO, Bresalier R, Andriole GL, Buys SS, Crawford ED, et al: Colorectal-cancer incidence and mortality with screening flexible sigmoidoscopy. N Engl J Med 366: 2345-2357, 2012.

7. Hirst Y, Kerrison R, Kobayashi LC, Counsell N, Djedovic N, Ruwende J, Stewart M and von Wagner C: Text Reminders in Colorectal Cancer Screening (TRICCS): Protocol for a randomised controlled trial. BMC Public Health 16: 74, 2016.

8. Miles A, Cockburn J, Smith RA and Wardle J: A perspective from countries using organized screening programs. Cancer 101 (Suppl 5): S1201-S1213, 2004.

9. Karsa LV, Lignini TA, Patnick J, Lambert R and Sauvaget C: The dimensions of the CRC problem. Best Pract Res Clin Gastroenterol 24: 381-396, 2010.

10. World Bank Country and Lending Groups for July 2016, available at https://datahelpdesk.worldbank.org/knowledgebase/articles/906519. Accessed March 23, 2018.

11. Mathers CD, Fat DM, Inoue M, Rao C and Lopez AD: Counting the dead and what they died from: An assessment of the global status of cause of death data. Bull World Health Organ 83: 171-177, 2005.

12. Ferlay J, Shin HR, Bray F, Forman D, Mathers C and Parkin DM: Estimates of worldwide burden of cancer in 2008: GLOBOCAN 2008. Int J Cancer 127: 2893-2917, 2010.

13. Altobelli E, Rapacchietta L, Angeletti PM, Barbante L, Profeta FV and Fagnano R: Breast cancer screening programmes across the WHO European region: Differences among countries based on National Income Level. Int J Environ Res Public Health 14: E452, 2017.

14. GBD 2013 Mortality and Causes of Death Collaborators: Global, regional, and national age-sex specific all-cause and cause-specific mortality for 240 causes of death, 1990-2013: A systematic analysis for the global burden of disease study 2013. Lancet 385: 117-171, 2015

15. Bray F, Jemal N, Grey N, Ferlay J and Forman D: Global cancer transitions according to the human development index (2008-2030): A population-based study. Lancet Oncol 13: 790-801, 2012.

16. Karim-Kos HE, de Vries E, Soerjomataram I, Lemmens V, Siesling S and Coebergh JW: Recent trends of cancer in Europe: A combined approach of incidence, survival and mortality for 17 cancer sites since the 1990s. Eur J Cancer 44: 1345-1389, 2008.

17. Altobelli E: Improving cervical cancer screening in Baltic, central, and eastern European countries. Lancet Oncol 17: 1349-1350, 2016.

18. Altobelli E and Lattanzi A: Cervical carcinoma in the European Union: An update on disease burden, screening program state of activation, and coverage as of March 2014. Int J Gynecol Cancer 25: 474-483, 2015.

19. Altobelli E and Lattanzi A: Breast cancer in European Union: An update of screening programmes as of March 2014 (Review). Int J Oncol 45: 1785-1792, 2014.

20. Global Burden of Disease Cancer Collaboration; Fitzmaurice C, Dicker D, Pain A, Hamavid H, Moradi-Lakeh M, MacIntyre MF, Allen C, Hansen G, Woodbrook R, Wolfe C, et al: The Global burden of cancer 2013. JAMA Oncol 1: 505-527, 2015.

21. Kuntz KM, Lansdorp-Vogelaar I, Rutter CM, Knudsen AB, van Ballegooijen M, Savarino JE, Feuer EJ and Zauber AG: A systematic comparison of microsimulation models of colorectal cancer: The role of assumptions about adenoma progression. Med Decis Making 31: 530-539, 2011. 
22. Brenner H, Hoffmeister M, Stegmaier C, Brenner G, Altenhofen L and Haug U: Risk of progression of advanced adenomas to colorectal cancer by age and sex: Estimates based on 840,149 screening colonoscopies. Gut 56: 1585-1589, 2007.

23. Schreuders EH, Ruco A, Rabeneck L, Schoen RE, Sung JJ, Young GP and Kuipers EJ: Colorectal cancer screening: A global overview of existing programmes. Gut 64: 1637-1649, 2015.

24. Lansdorp-VogelaarI,Knudsen AB andBrennerH:Cost-effectiveness of colorectal cancer screening. Epidemiol Rev 33: 88-100, 2011.

25. Ginsberg GM, Lauer JA, Zelle S, Baeten S and Baltussen R: Cost effectiveness of strategies to combat breast, cervical, and colorectal cancer in sub-Saharan Africa and South East Asia: Mathematical modelling study. BMJ 344: e614, 2012.

26. von Karsa L, Patnick J and Segnan N: European guidelines for quality assurance in colorectal cancer screening and diagnosis. First Edition-Executive summary. Endoscopy 44 (Suppl 3): SE1-SE8, 2012.
27. Moss S, Ancelle-Park R and Brenner H; International Agency for Research on Cancer: European guidelines for quality assurance in colorectal cancer screening and diagnosis. First Edition-Evaluation and interpretation of screening outcomes. Endoscopy 44 (Suppl 3): SE49-SE64, 2012.

28. Forman D, Bray F, Brewster DH, Gombe Mbalawa C, Kohler B, Piñeros M, Steliarova-Foucher E and Swaminathan R: Cancer Incidence in Five Continents. Vol 10. IARC Press, Lyon, France, 2014.

29. Bray F, Znaor A, Cueva P, Korir A, Swaminathan R, Ullrich A, Wang SA and Parkin DM: Planning and Developing Population-based Cancer Registration in Low- and Middle-income Settings. IARC Technical publication No. 43. IARC Press, Lyon, France, 2014. 